Commun. Korean Math. Soc. 21 (2006), No. 4, pp. 689-700

\title{
GENERALIZED NONLINEAR MULTIVALUED MIXED QUASI-VARIATIONAL-LIKE INEQUALITIES
}

\author{
Byung-Soo Lee, M. Firdosh Khan, and Salahuddin
}

\begin{abstract}
In this paper, we introduce a new class of generalized nonlinear multivalued mixed quasi-variational-like inequalities and prove the existence and uniqueness of solutions for the class of generalized nonlinear multivalued mixed quasi-variational-like inequalities in reflexive Banach spaces using Fan-KKM Theorem.
\end{abstract}

\section{Introduction and preliminaries}

Variational inequality theory, which constituted a significant extension of the variational principle, has described a broad spectrum of very interesting developments involving a link among various fields of mathematics, physics, economics, regional and engineering sciences. The ideas and techniques are being applied in a variety of diverse areas of sciences and prove to be productive and innovative. Hence variational inequality theory has become a very effective and powerful tool for studying a wide range of problems arising in pure and applied sciences which include work on differential equations, mechanics, contact problems in elasticity, control problems, general equilibrium problems in economics and transportation, and unilateral, obstacle, optimization, etc. $[1,2,3,7,8,11,12,17,18]$. Among many kinds of variational inequalities, variational-like inequalities were firstly posed by Parida and Sen [13] and quasi-variational inequalities were firstly introduced by Aubin and Ekeland [1]. In fact, the quasi-variational-like inequality, which is an extension and a generalization of the variational inequalities mentioned above, is desirable to be considered more deeply.

Received March 17, 2006.

2000 Mathematics Subject Classification: 49J40, 90C33.

Key words and phrases: nonlinear multivalued mixed quasi-variational-like inequalities, monotone type mappings, generalized Lipschitz type mapping, KKMmapping. 
On the other hand, recently a new class of monotone nonlinear variational inequalities was introduced and the existence of solutions to them was considered in [16].

In this paper, a new class of generalized nonlinear multivalued mixed quasi-variational-like inequalities are introduced and studied. Also the solvability of this class of generalized nonlinear multivalued mixed quasivariational-like inequalities in reflexive Banach spaces is given. Our results improve and extend the corresponding results of $[6,10,14,15,16]$.

Throughout this paper, $X$ is a real Banach space with dual space $X^{\star}$ and $K$ is a nonempty convex closed subset of $X$. Denote $\langle\ell, x\rangle=\ell(x)$, for all $\ell \in X^{\star}$ and $x \in X$. Let $S, T: K \rightarrow 2^{X^{\star}}$ be two multivalued mappings, $N: X^{\star} \times X^{\star} \rightarrow X^{\star}$ and $g: K \rightarrow X^{\star}$ be mappings. Let a mapping $\eta: K \times K \rightarrow K$ be affine with respect to the first argument satisfying $\eta(u, v)=-\eta(v, u)$ for all $u, v \in K$. We consider the following generalized nonlinear multivalued mixed quasi-variational-like inequality problem (in short, GNMMQVLIP):

For any $\ell \in X^{\star}$, find $u \in K$ such that

$$
\sup _{x \in S(u), y \in T(u)}\langle(g(u)+N(x, y))-\ell, \eta(v, u)\rangle+f(v)-f(u) \geq 0 \text { for all } v \in K,
$$

where $f: K \rightarrow \mathbb{R} \cup\{+\infty\}$ be a proper convex functional.

We note that, if $g$ is a zero mapping then (1) is reduced to the following problem:

For any $\ell \in X^{\star}$, find $u \in K$ such that

$$
\sup _{x \in S(u), y \in T(u)}\langle N(x, y)-\ell, \eta(v, u)\rangle+f(v)-f(u) \geq 0 \text { for all } v \in K,
$$

which is called a generalized nonlinear multivalued quasi-variational-like inequality problem.

We remark that if $\eta(v, u)=v-u$, then (2) is collapsed to the following problem:

For any $\ell \in X^{\star}$, find $u \in K$ such that

$$
\sup _{x \in S(u), y \in T(u)}\langle N(x, y)-\ell, v-u\rangle+f(v)-f(u) \geq 0 \text { for all } v \in K,
$$

which was studied by Cho et al. [4].

We note that, if $N(x, y)=x-y$, then (2) is equivalent to following problem:

For any $\ell \in X^{\star}$, find $u \in K$ such that

$$
\sup _{x \in S(u), y \in T(u)}\langle x-y-\ell, \eta(v, u)\rangle+f(v)-f(u) \geq 0 \text { for all } v \in K,
$$


which was considered by Fang et al. [6].

If $S$ and $T$ are single-valued mappings, then (2) is equivalent to following problem:

For any $\ell \in X^{\star}$, find $u \in K$ such that

$$
\langle N(S(u), T(u))-\ell, \eta(v, u)\rangle+f(v)-f(u) \geq 0 \text { for all } v \in K,
$$

which was studied by Huang et al. [10].

We remark that if $\eta(v, u)=G(v)-G(u)$, where $G: K \rightarrow K$ is a mapping, then (1) is collapsed to the following problem:

For any $\ell \in X^{\star}$, find $u \in K$ such that

$$
\sup _{x \in S(u), y \in T(u)}\langle(g(u)+N(x, y))-\ell, G(v)-G(u)\rangle+f(v)-f(u) \geq 0
$$

for all $v \in K$.

Again, we note that if $G$ is an identity mapping, then $G(v)-G(u)=$ $v-u$, therefore (6) is collapsed to the following problem:

For any $\ell \in X^{\star}$, find $u \in K$ such that

$$
\sup _{x \in S(u), y \in T(u)}\langle(g(u)+N(x, y))-\ell, v-u\rangle+f(v)-f(u) \geq 0 \text { for all } v \in K .
$$

REMARK 1.1. For a suitable choice of $g, N, S, T, \eta$ and $f$, the problem (1) includes many known variational inequalities as special cases (see $[4,6,10,15,16]$ and the references therein).

In the sequel, we recall some definitions needed.

Definition 1.1. A mapping $S: K(\subset X) \rightarrow 2^{X^{\star}}$ is said to be $\eta$ - $\varphi$-p-monotone with respect to the first argument of a mapping $N$ : $X^{\star} \times X^{\star} \rightarrow X^{\star}$ if there exist a function $\varphi:[0,+\infty) \rightarrow[0,+\infty)$, a mapping $\eta: K \times K \rightarrow K$ and a constant $p>1$ such that

$$
\langle N(x, \cdot)-N(y, \cdot), \eta(u, v)\rangle \geq \varphi(\|\eta(u, v)\|)\|\eta(u, v)\|^{p}
$$

for all $u, v \in K, x \in S(u)$ and $y \in S(v)$.

Definition 1.2. A mapping $T: K(\subset X) \rightarrow 2^{X^{\star}}$ is said to be $\eta$ $\psi$-p-monotone with respect to the second argument of a mapping $N$ : $X^{\star} \times X^{\star} \rightarrow X^{\star}$ if there exist a function $\psi:[0,+\infty) \rightarrow[0,+\infty)$, a mapping $\eta: K \times K \rightarrow K$ and a constant $p>1$ such that

$$
\langle N(\cdot, x)-N(\cdot, y), \eta(u, v)\rangle \geq-\psi(\|\eta(u, v)\|)\|\eta(u, v)\|^{p}
$$

for all $u, v \in K, x \in T(u)$ and $y \in T(v)$. 
Definition 1.3. A mapping $g: K(\subset X) \rightarrow X^{\star}$ is said to be $\eta$ - $\phi$ - $p$ relaxed Lipschitzian if there exist a function $\phi:[0,+\infty) \rightarrow[0,+\infty)$, a mapping $\eta: K \times K \rightarrow K$ and a constant $p>1$ such that

$$
\langle g(v)-g(u), \eta(v, u)\rangle \geq \phi(\|\eta(v, u)\|)\|\eta(v, u)\|^{p} \text { for all } u, v \in K .
$$

REMARK 1.2. For a suitable choice of $N, S, T, g$ and $\eta$ we will provide many concepts which are special cases of our concepts (see [4, $6,9,10,14,16]$ and the references therein).

Definition 1.4. Let $X$ and $Y$ be topological spaces. A mapping $F: X \rightarrow 2^{Y}$ is said to be lower semi-continuous at $x \in X$ if for any $y \in F(x)$ and for any net $\left\{x_{\alpha}\right\}$ in $X$ converging to $x$, there exists a subset $\left\{x_{\beta}\right\} \subset\left\{x_{\alpha}\right\}$ and $y_{\beta} \in F\left(x_{\beta}\right)$ for each $\beta$ such that $\left\{y_{\beta}\right\}$ converging to $y$.

Definition 1.5 [14]. A mapping $g: K \rightarrow X^{\star}$ is said to be hemicontinuous if for all $u, v, z \in K$, the mapping $t \rightarrow\langle g(u+t v), z\rangle$ is continuous on [0,1]. A mapping $T: K \rightarrow 2^{X^{\star}}$ is said to be lower hemicontinuous if for all $u, v, z \in K$, the multivalued mapping

$$
t \rightarrow\langle T(u+t v), z\rangle
$$

is lower semi-continuous on $[0,1]$.

\section{Main results}

Now, we give our main results in this paper.

TheOREM 2.1. Let $X$ be a reflexive Banach space, $X^{\star}$ be its dual and $K$ be a nonempty convex closed subset of $X$, let $g: K \rightarrow X^{\star}$ be a hemicontinuous mapping satisfying (10) and also let $S$ and $T: K \rightarrow 2^{X^{\star}}$ be lower semi-continuous multivalued mappings satisfying (8) and (9), respectively, where for functions $\varphi, \psi, \phi:[0,+\infty) \rightarrow[0,+\infty)$ satisfying $\varphi(t)+\phi(t)>\psi(t)$ for all $t>0, \varphi+\phi-\psi$ is bounded in $[0, \delta]$ for some $\delta>0$. In addition, suppose that $\eta(u, v)=-\eta(v, u)$ for all $u, v \in K$, $\eta: K \times K \rightarrow K$ is affine with respect to the first argument, $f: K \rightarrow$ $\mathbb{R} \cup\{+\infty\}$ is a proper convex functional and $N: X^{\star} \times X^{\star} \rightarrow X^{\star}$ is continuous with respect to the weak $k^{\star}$ topology of $X$. Let a multivalued mapping $v \mapsto\left\{N(z, w) \in X^{\star}: z \in S(v), w \in T(v)\right\}$ be lower hemicontinuous. Then for any $\ell \in X^{\star}, u \in K$ is a solution of problem (1) if and only if $u \in K$ is a solution of the following problem: 
Find $u \in K$ such that

$$
\begin{aligned}
& \langle(g(v)+N(z, w))-\ell, \eta(v, u)\rangle+f(v)-f(u) \\
\geq & (\varphi(\|\eta(v, u)\|)-\psi(\|\eta(v, u)\|)+\phi(\|\eta(v, u)\|))\|\eta(v, u)\|^{p}
\end{aligned}
$$

for all $v \in K, z \in S(v)$ and $w \in T(v)$.

Proof. Suppose that the problem (1) holds. Since mappings $S, T$ and $g$ satisfy (8), (9) and (10), respectively, then for all $u, v \in K, x \in$ $S(u), z \in S(v), y \in T(u)$ and $w \in T(v)$, we have

$$
\begin{aligned}
& \langle(g(v)+N(z, w))-\ell, \eta(v, u)\rangle+f(v)-f(u) \\
= & \langle-\ell, \eta(v, a u)\rangle+\langle g(v), \eta(v, u)\rangle+\langle N(z, w), \eta(v, u)\rangle+f(v)-f(u) \\
= & \langle-\ell, \eta(v, u)\rangle-\langle N(x, w)-N(z, w), \eta(v, u)\rangle \\
& -\langle N(x, y)-N(x, w), \eta(v, u)\rangle \\
& +\langle N(x, y), \eta(v, u)\rangle+\langle g(v)-g(u), \eta(v, u)\rangle \\
& +\langle g(u), \eta(v, u)\rangle+f(v)-f(u) \\
= & \langle-\ell, \eta(v, u)\rangle+\langle N(x, w)-N(z, w), \eta(u, v)\rangle \\
& +\langle N(x, y)-N(x, w), \eta(u, v)\rangle+\langle N(x, y), \eta(v, u)\rangle \\
& +\langle g(v)-g(u), \eta(v, u)\rangle+\langle g(u), \eta(v, u)\rangle+f(v)-f(u) \\
\geq & \langle(g(u)+N(x, y))-\ell, \eta(v, u)\rangle+f(v)-f(u)+(\varphi(\|\eta(v, u)\|) \\
& -\psi(\|\eta(v, u)\|)+\phi(\|\eta(v, u)\|))\|\eta(v, u)\|^{p} .
\end{aligned}
$$

Put $A=\langle(g(v)+N(z, w))-\ell, \eta(v, u)\rangle+f(v)-f(u)$

$B=\langle(g(u)+N(x, y))-\ell, \eta(v, u)\rangle+f(v)-f(u)$

$C=(\varphi-\psi+\phi)(\|\eta(v, u)\|)\|\eta(v, u)\|^{p}$.

Taking suprema on both sides of the following inequality;

$$
A \geq B+C,
$$

we have

$$
\begin{gathered}
A=\sup _{x \in S(u), y \in T(u)} A \geq \sup _{x \in S(u), y \in T(u)}(B+C)=\sup _{x \in S(u), y \in T(u)} B+C . \\
\text { Since } \sup _{x \in S(u), y \in T(u)} B \geq 0 \text { for all } v \in K \text { from }(1), A \geq C \text {. Hence } \\
\langle(g(v)+N(z, w))-\ell, \eta(v, u)\rangle+f(v)-f(u) \\
\geq(\varphi(\|\eta(v, u)\|)-\psi(\|\eta(v, u)\|)+\phi(\|\eta(v, u)\|))\|\eta(v, u)\|^{p}
\end{gathered}
$$

for all $v \in K, z \in S(v)$ and $w \in T(v)$, i.e., (11) is true.

Conversely, suppose that (11) holds, without loss of generality, choose a point $v \in K$ such that $f(v)<+\infty$ and so $f(u)<+\infty$. Letting $v_{n}=$ 
$\left(1-\frac{1}{n}\right) u+\frac{1}{n} v$ for $n \in \mathbb{N}$, we have $v_{n} \in K$. Since $\eta: K \times K \rightarrow K$ is affine with respect to the first argument and $\eta(u, u)=0, \eta\left(v_{n}, u\right)=\frac{1}{n} \eta(v, u)$ for $n \in \mathbb{N}$.

For any $x \in S(u)$ and $y \in T(u)$, since the mapping $v \mapsto\{N(z, w) \in$ $\left.X^{\star}: z \in S(v), w \in T(v)\right\}$ is lower hemi-continuous, the mapping $v \mapsto g(v)$ is hemi-continuous and $v_{n} \rightarrow u$ as $n \rightarrow \infty$, there exists a subsequence $\left\{v_{n_{j}}\right\} \subset\left\{v_{n}\right\}$ and there are $z_{n_{j}} \in S\left(v_{n_{j}}\right), w_{n_{j}} \in T\left(v_{n_{j}}\right)$ such that for any $\tau \in X$

$(12) z_{n_{j}} \rightarrow x, w_{n_{j}} \rightarrow y,\left\langle g\left(v_{n_{j}}\right)+N\left(z_{n_{j}}, w_{n_{j}}\right), \tau\right\rangle \rightarrow\langle g(u)+N(x, y), \tau\rangle$

as $j \rightarrow \infty$. It follows from (11) that

$$
\begin{aligned}
& \left\langle g\left(v_{n_{j}}\right)+N\left(z_{n_{j}}, w_{n_{j}}\right)-\ell, \eta\left(v_{n_{j}}, u\right)\right\rangle+f\left(v_{n_{j}}\right)-f(u) \\
\geq & \left(\varphi\left(\left\|\eta\left(v_{n_{j}}, u\right)\right\|\right)-\psi\left(\left\|\eta\left(v_{n_{j}}, u\right)\right\|\right)+\phi\left(\left\|\eta\left(v_{n_{j}}, u\right)\right\|\right)\right)\left\|\eta\left(v_{n_{j}}, u\right)\right\|^{p} \\
= & \left(\frac{1}{n_{j}}\right)^{p}\left(\varphi\left(\frac{1}{n_{j}}\|\eta(v, u)\|\right)-\psi\left(\frac{1}{n_{j}}\|\eta(v, u)\|\right)\right. \\
& \left.+\phi\left(\frac{1}{n_{j}}\|\eta(v, u)\|\right)\right)\|\eta(v, u)\|^{p},
\end{aligned}
$$

since $\eta\left(v_{n_{j}}, u\right)=\frac{1}{n_{j}} \eta(v, u)$.

Since $f$ is convex and $v_{n_{j}}=\left(1-\frac{1}{n_{j}}\right) u+\frac{1}{n_{j}} v$,

$$
\begin{aligned}
f(v)-\dot{f}(u) & =n_{j}\left(\left(1-\frac{1}{n_{j}}\right) f(u)+\frac{1}{n_{j}} f(v)-f(u)\right) \\
& \geq n_{j} f\left(\left(1-\frac{1}{n_{j}}\right) u+\frac{1}{n_{j}} v\right)-n_{j} f(u) \\
& =n_{j} f\left(v_{n_{j}}\right)-n_{j} f(u) \\
& =n_{j}\left(f\left(v_{n_{j}}\right)-f(u)\right),
\end{aligned}
$$

from (13) it follows that

$$
\begin{aligned}
& \left\langle g\left(v_{n_{j}}\right)+N\left(z_{n_{j}}, w_{n_{j}}\right)-\ell, \eta(v, u)\right\rangle+f(v)-f(u) \\
\geq & \left(\frac{1}{n_{j}}\right)^{p-1}\left(\varphi\left(\frac{1}{n_{j}}\|\eta(v, u)\|\right)-\psi\left(\frac{1}{n_{j}}\|\eta(v, u)\|\right)\right. \\
+ & \left.\phi\left(\frac{1}{n_{j}}\|\eta(v, u)\|\right)\right)\|\eta(v, u)\|^{p} .
\end{aligned}
$$

It follows from (12) and (14) that

$$
\langle(g(u)+N(x, y)-\ell, \eta(v, u)\rangle+f(v)-f(u) \geq 0
$$

for all $v \in K, x \in S(u), y \in T(u)$. This completes the proof. 
REMARK 2.1. Theorem 2.1 improves and extends Theorem 2.1 of $[4,5,6,10,16]$.

From Theorem 2.1, we have the following theorem.

Corollary 2.2. Let $G: K \rightarrow K$ be an affine mapping and put $\eta(v, u)=G(v)-G(u)$ for $u, v \in K$ in Definition 2.1, Definition 2.2, Definition 2.3 and Theorem 2.1. Then for any $\ell \in X^{\star}, u \in K$ is a solution of the problem (6) if and only if $u \in K$ is a solution of the following problem.

For any $\ell \in X^{\star}$, find $u \in K$ such that

$$
\begin{aligned}
& \langle(g(v)+N(z, w))-\ell, G(v)-G(u)\rangle+f(v)-f(u) \\
\geq & (\varphi(\|G(v)-G(u)\|)-\psi(\|G(v)-G(u)\|) \\
& +\phi(\|G(v)-G(u)\|))\|G(v)-G(u)\|^{p}
\end{aligned}
$$

for all $v \in K, z \in S(v)$ and $w \in T(v)$.

From Corollary 2.2, we obtain the main result Theorem 2.1 of Cho et al. [4] as a corollary.

Corollary 2.3 [4]. Let $G$ be an identity mapping, $g \equiv 0, \phi \equiv 0$ and $N(x, y)=x-y$ for $x, y \in X^{\star}$ in Corollary 2.2. Then for any $\ell \in X^{\star}$, $u \in K$ is a solution of

$$
\sup _{x \in S(u), y \in T(u)}\langle N(x, y)-\ell, v-u\rangle+f(v)-f(u) \geq 0 \quad \text { for all } v \in K
$$

if and only if $u \in K$ is a solution of

$$
\langle N(z, w)-\ell, v-u\rangle+f(v)-f(u) \geq(\varphi\|v-u\|)-\psi(\|v-u\|)\|v-u\|^{p}
$$

for all $v \in K, z \in S(v)$ and $w \in T(v)$.

The following definition and result are essential for our further result.

Definition 2.1 [17]. Let $X$ be a topological vector space. A mapping $F: X \rightarrow 2^{X}$ is called a KKM mapping if for any $\left\{x_{1}, x_{2}, \ldots, x_{n}\right\} \subset$ $X$,

$$
c o\left\{x_{1}, x_{2}, \ldots, x_{n}\right\} \subset \bigcup_{i=1}^{n} F\left(x_{i}\right) .
$$

FAN-KKM THEOREM 2.4 [5]. Let $K$ be a nonempty subset of a topological vector space $X$ and $F: K \rightarrow 2^{X}$ be a KKM-mapping. If $F(x)$ is closed in $X$ for every $x$ in $K$ and there exists at least a point $x_{0} \in K$ such that $F\left(x_{0}\right)$ is compact, then

$$
\bigcap_{x \in K} F(x) \neq \emptyset
$$


THeOREM 2.5. Let $X$ be a real reflexive Banach space, $X^{\star}$ be its dual space and $K$ be a nonempty bounded closed convex subset of $X$. Let $S, T, g, N, \varphi, \psi, \phi$ and $\eta$ be the same as those in Theorem 2.1. Suppose that the mapping $\eta$ and a function $\varphi-\psi+\phi$ are continuous and $f$ : $K \rightarrow \mathbb{R} \cup\{+\infty\}$ is a proper convex lower semi-continuous. Then the problem (1) has a solution. Moreover if $\eta(u, v)=0$ implies $u=v$, then the problem (1) has a unique solution.

Proof. We first prove the existence of a solution of the problem (1). Define two multivalued mappings $F, H: K \rightarrow 2^{K}$ by, for any $\ell \in X^{\star}$,

$$
\begin{aligned}
F(v)=\{u \in K:\langle(g(u)+N(x, y))-\ell, \eta(v, u)\rangle+f(v)-f(u) \geq 0 \\
\\
\quad \text { for some } x \in S(u), y \in T(u)\}
\end{aligned}
$$

for all $v \in K$, and

$$
\begin{aligned}
H(v)= & \{u \in K:\langle(g(v)+N(z, w))-\ell, \eta(v, u)\rangle+f(v)-f(u) \\
& \geq(\varphi(\|\eta(v, u)\|)-\psi(\|\eta(v, u)\|)+\phi(\|\eta(v, u)\|))\|\eta(v, u)\|^{p} \\
& \text { for all } z \in S(v), w \in T(v)\}
\end{aligned}
$$

for all $v \in K$.

We show that $F$ is a KKM-mapping. Assume that $F$ is not a KKMmapping. Then there exists $\left\{v_{1}, v_{2}, \ldots, v_{n}\right\} \subset K$ and $t_{i}>0, i=$ $1,2, \ldots, n$, such that

$$
\sum_{i=1}^{n} t_{i}=1, v=\sum_{i=1}^{n} t_{i} v_{i} \notin \bigcup_{i=1}^{n} F\left(v_{i}\right) .
$$

For any $z \in S(u)$ and $w \in T(u)$, by the definition of $F$, we have

$$
\left\langle(g(v)+N(z, w))-\ell, \eta\left(v_{i}, v\right)\right\rangle+f\left(v_{i}\right)-f(v)<0
$$

for $i=1,2, \ldots, n$. It follows that

$$
\begin{aligned}
0 & =\langle(g(v)+N(z, w))-\ell, \eta(v, v)\rangle \\
& =\left\langle(g(v)+N(z, w))-\ell, \eta\left(\sum_{i=1}^{n} t_{i} v_{i}, v\right)\right\rangle \\
& =\sum_{i=1}^{n} t_{i}\left\langle(g(v)+N(z, w))-\ell, \eta\left(v_{i}, v\right)\right\rangle<\sum_{i=1}^{n} t_{i}\left(f(v)-f\left(v_{i}\right)\right) \\
& =f(v)-\sum_{i=1}^{n} t_{i} f\left(v_{i}\right) \leq f(v)-f(v)=0
\end{aligned}
$$

which is a contradiction. This implies that $F$ is a KKM-mapping. Now we prove that $F(v) \subset H(v)$ for all $v \in K$. Let $u \in F(v)$. Then there exist $x \in S(u), y \in T(u)$ such that

$$
\langle(g(u)+N(x, y))-\ell, \eta(v, u)\rangle+f(v)-f(u) \geq 0 .
$$


Since the mappings $g, S$ and $T$ satisfy (10), (8) and (9), respectively, we have

$$
\begin{aligned}
& \langle(g(v)+N(z, w))-\ell, \eta(v, u)\rangle+f(v)-f(u) \\
= & \langle-\ell, \eta(v, u)\rangle+\langle N(x, w)-N(z, w), \eta(u, v)\rangle \\
& +\langle N(x, y)-N(x, w), \eta(u, v)\rangle+\langle N(x, y), \eta(v, u)\rangle \\
& +\langle g(v)-g(u), \eta(v, u)\rangle+\langle g(u), \eta(v, u)\rangle+f(v)-f(u) \\
\geq & (\varphi(\|\eta(v, u)\|)-\psi(\|\eta(v, u)\|)+\phi(\|\eta(v, u)\|))\|\eta(v, u)\|^{p} \\
& +\langle(g(u)+N(x, y)-\ell, \eta(v, u)\rangle+f(v)-f(u) \\
\geq & (\varphi(\|\eta(v, u)\|)-\psi(\|\eta(v, u)\|)+\phi(\|\eta(v, u)\|))\|\eta(v, u)\|^{p}
\end{aligned}
$$

for all $v \in K, z \in S(v)$ and $w \in T(v)$. This implies that $u \in H(v)$ and so $H$ is also a KKM-mapping.

From the assumption, it follows that $H(v)$ is weakly closed for all $v \in K$. Since $K$ is bounded closed convex, we know that $K$ is weakly compact and so $H(v)$ is weakly compact in $K$ for all $v \in K$. It follows from Fan-KKM Theorem that

$$
\bigcap_{v \in K} H(v) \neq \emptyset
$$

Hence for any $\ell \in X^{\star}$ there exists a point $u_{0} \in K$ such that

$$
\begin{aligned}
& \left\langle(g(v)+N(z, w))-\ell, \eta\left(v, u_{0}\right)\right\rangle+f(v)-f\left(u_{0}\right) \\
\geq & \left(\varphi\left(\left\|\eta\left(v, u_{0}\right)\right\|\right)-\psi\left(\left\|\eta\left(v, u_{0}\right)\right\|\right)+\phi\left(\left\|\eta\left(v, u_{0}\right)\right\|\right)\right)\left\|\eta\left(v, u_{0}\right)\right\|^{p}
\end{aligned}
$$

for all $z \in S(v), w \in T(v)$ for all $v \in K$. Thus

$$
\left\langle\left(g\left(u_{0}\right)-N(x, y)\right)-\ell, \eta\left(v, u_{0}\right)\right\rangle+f(v)-f\left(u_{0}\right) \geq 0,
$$

for all $v \in K$, for some $x \in S(v)$ and for some $y \in T(v)$, which shows that $u_{0}$ is a solution of (1).

Let $u_{1}$ and $u_{2} \in K$ be solutions of the problem (1). Since

$$
k_{1}(v):=\sup _{\substack{x \in S\left(u_{1}\right) \\ y \in T\left(u_{1}\right)}}\left\langle\left(g\left(u_{1}\right)+N(x, y)\right)-\ell, \eta\left(v, u_{1}\right)\right\rangle+f(v)-f\left(u_{1}\right) \geq 0
$$

for all $v \in K$ and

$$
k_{2}(v):=\sup _{\substack{x \in S\left(u_{2}\right) \\ y \in T\left(u_{2}\right)}}\left\langle\left(g\left(u_{2}\right)+N(x, y)\right)-\ell, \eta\left(v, u_{2}\right)\right\rangle+f(v)-f\left(u_{2}\right) \geq 0
$$

for all $v \in K$, 
by the definition of supremum, for any positive number $\varepsilon$, there exist $x_{1} \in S\left(u_{1}\right)$ and $y_{1} \in T\left(u_{1}\right)$ such that

(16) $k_{1}(v)-\varepsilon<\left\langle\left(g\left(u_{1}\right)+N\left(x_{1}, y_{1}\right)\right)-\ell, \eta\left(v, u_{1}\right)\right\rangle+f(v)-f\left(u_{1}\right) \leq k_{1}(v)$ and, there exist $x_{2} \in S\left(u_{2}\right)$ and $y_{2} \in T\left(u_{2}\right)$ such that

Setting $v=u_{2}$ in (16) and $v=u_{1}$ in (17) and adding, we have

$$
\begin{aligned}
& k_{1}\left(u_{2}\right)+k_{2}\left(u_{1}\right)-2 \varepsilon \\
< & \left\langle g\left(u_{1}\right)-g\left(u_{2}\right)+N\left(x_{1}, y_{1}\right)-N\left(x_{2}, y_{2}\right), \eta\left(u_{2}, u_{1}\right)\right\rangle \\
\leq & k_{1}\left(u_{2}\right)+k_{2}\left(u_{1}\right) .
\end{aligned}
$$

Since $\varepsilon$ is arbitrary,

$$
\begin{aligned}
& \left\langle g\left(u_{1}\right)-g\left(u_{2}\right)+N\left(x_{1}, y_{1}\right)-N\left(x_{2}, y_{2}\right), \eta\left(u_{2}, u_{1}\right)\right\rangle \\
= & k_{1}\left(u_{2}\right)+k_{2}\left(u_{1}\right) \geq 0 .
\end{aligned}
$$

By (8), (9) and (10), we obtain

$$
\begin{aligned}
& \left\langle g\left(u_{1}\right)-g\left(u_{2}\right)+N\left(x_{1}, y_{1}\right)-N\left(x_{2}, y_{2}\right), \eta\left(u_{2}, u_{1}\right)\right\rangle \\
= & \left\langle g\left(u_{1}\right)-g\left(u_{2}\right), \eta\left(u_{2}, u_{1}\right)\right\rangle+\left\langle N\left(x_{1}, y_{1}\right)-N\left(x_{2}, y_{1}\right), \eta\left(u_{2}, u_{1}\right)\right\rangle \\
& +\left\langle N\left(x_{2}, y_{1}\right)-N\left(x_{2}, y_{2}\right), \eta\left(u_{2}, u_{1}\right)\right\rangle \\
= & -\left\langle g\left(u_{1}\right)-g\left(u_{2}\right), \eta\left(u_{1}, u_{2}\right)\right\rangle-\left\langle N\left(x_{1}, y_{1}\right)-N\left(x_{2}, y_{1}\right), \eta\left(u_{1}, u_{2}\right)\right\rangle \\
& -\left\langle N\left(x_{2}, y_{1}\right)-N\left(x_{2}, y_{2}\right), \eta\left(u_{1}, u_{2}\right)\right\rangle \\
\leq & \left(-\phi\left(\left\|\eta\left(u_{1}, u_{2}\right)\right\|\right)-\varphi\left(\left\|\eta\left(u_{1}, u_{2}\right)\right\|\right)+\psi\left(\left\|\eta\left(u_{1}, u_{2}\right)\right\|\right)\right)\left\|\eta\left(u_{1}, u_{2}\right)\right\|^{p} \\
= & -\left(\phi\left(\left\|\eta\left(u_{1}, u_{2}\right)\right\|\right)+\varphi\left(\left\|\eta\left(u_{1}, u_{2}\right)\right\|\right)-\psi\left(\left\|\eta\left(u_{1}, u_{2}\right)\right\|\right)\right)\left\|\eta\left(u_{1}, u_{2}\right)\right\|^{p} .
\end{aligned}
$$

Due to the inequality $\phi(t)+\varphi(t)>\psi(t)$ for all $t>0$, it follows from (8) and (9) that $\left\|\eta\left(u_{1}, u_{2}\right)\right\|^{p}=0$.

By the assumption that $\eta(u, v)=0$ implies $u=v$, we have $u_{1}=u_{2}$. This completes the proof.

REMark 2.2. Theorem 2.4 also improves and extends Theorem 2.4 of $[4,6,10,16]$.

\section{References}

[1] J. P. Aubin and I. Ekeland, Applied Nonlinear Analysis, John Wiley \& Sons, Inc., New York, 1984.

[2] F. E. Browder, On the unification of the calculus of variations and the theory of monotone nonlinear operators in Banach spaces, Proc. Nat. Acad. Sci. U.S.A 56 (1966), 419-425. 
[3] F. E. Browder and W. V. Petryshyn, Construction of fixed points of nonlinear mappings in Hilbert spaces, J. Math. Anal. Appl. 20 (1967), 197-228.

[4] Y. J. Cho, Y. P. Fang, N. J. Huang, and K. H. Kim, Generalized set valued strongly nonlinear variational inequalities in Banach spaces, J. Korean Math. Soc. 40 (2003), no. 2, 195-205.

[5] K. Fan, Some properties of convex sets related to fixed points theorem, Math. Annal. 266 (1984), 519-537.

[6] Y. P. Fang, Y. J. Cho, N. J. Huang, and S. M. Kang, Generalized nonlinear quasivariational-like inequalities for set valued mappings in Banach spaces, Math. Inequal. Appl. 6 (2003), no. 2, 331-337.

[7] F. Giannessi and A. Maugeri, Variational Inequalities and Network Equilibrium Problems, Pleum, New York, 1995.

[8] P. T. Harker and J. S. Pang, Finite dimensional variational inequality and nonlinear complementarity problems; A survey of theory, algorithms and applications, Math. Program. 48 (1990), 161-220.

[9] N. J. Huang and C. X. Deng, Auxiliary principle and iterative algorithms for generalized set-valued strongly nonlinear mixed variational-like inequalities, $\mathrm{J}$. Math. Anal. Appl. 256 (2001), 345-359.

[10] N. J. Huang, Y. P. Fang, and Y.J. Cho, A new class of generalized nonlinear mixed quasi-variational inequalities in Banach spaces, Math. Inequal. Appl. 6 (2003), no. 1, 125-132.

[11] N. J. Huang, Y. P. Liu, Y. Y. Tang, and M. R. Bai, On the generalized setvalued strongly nonlinear implicit variational inequalities, Comput. Math. Appl. 37 (1999), no. 10, 29-36.

[12] P. D. Panagiotopoulos, Inequality Problems in Mechanics and Applications, Birkhauser, Boston, 1995.

[13] J. Parida and A. Sen, A variational-like inequality for multifunctions with applications, J. Math. Anal. Appl. 124 (1987), 73-81.

[14] R. U. Verma, Nonlinear variational inequalities on convex subsets of Banach spaces, Appl. Math. Lett. 10 (1997), no. 4, 25-27.

[15] _ Generalized pseudo-contractions and nonlinear variational inequalities, Publ. Math. Debrecen 53 (1998), no. 1-2, 23-28.

[16] __ On monotone nonlinear variational inequality problems, Comment Math. Univ. Carolinae 39 (1998), no. 1, 91-98.

[17] G. X. Z. Yuan, KKM Theory and Applications, Marcel Dekker, New York, 1999.

[18] E. Zeidler, Nonlinear Functional Analysis and its Applications, Springer-Verlag, New York, 1988.

Byung-Soo Lee

Department of Mathematics

Kyungsung University

Busan 608-736, Korea

E-mail: bslee@ks.ac.kr 
M. Firdosh Khan and Salahuddin

Department of Mathematics

Aligarh Muslim University, Aligarh-202002, India

E-mail: khan_mfk@yahoo.com 